\title{
TBS and BMD at the end of Al-therapy: a prospective study of the B-ABLE cohort.
}

AUTHORS: Rodríguez-Sanz María ${ }^{1}$, Pineda-Moncusí Marta ${ }^{1}$, Servitja Sonia ${ }^{2}$, Garcia-Giralt Natalia ${ }^{1 凶}$, Martos Tamara $^{2}$, Tusquets Ignasi ${ }^{2}$, Martínez-García Maria ${ }^{2}$, Rodriguez-Morera Jaime $^{3}$, Diez-Perez Adolfo ${ }^{1,3}$, Albanell Joan ${ }^{2}$, Nogués Xavier ${ }^{1,3}$

\section{AFFILIATIONS}

1. IMIM (Hospital del Mar Research Institute), Red Temática de Investigación Cooperativa en Envejecimiento y Fragilidad (RETICEF), Barcelona, Spain.

2. Cancer Research Program, IMIM (Hospital del Mar Research Institute), Medical Oncology Department, Hospital del Mar, Universitat Autònoma de Barcelona, Barcelona, Spain.

3. Internal Medicine Department, Hospital del Mar, Universitat Autònoma de Barcelona, Barcelona, Spain.

\section{${ }^{\square}$ CORRESPONDING AUTHOR:}

Dr Natalia Garcia Giralt

Hospital del Mar Medical Research Institute

PRBB

Barcelona, CP:08003 (Spain). 


\section{ABSTRACT}

$\underline{\text { Introduction }}$

Patients with breast cancer under aromatase inhibitor (Al) treatment often develop osteoporosis and their average bone loss rate is twice that of natural reduction during menopause, increasing fracture risk. As the current diagnostic technique based on bone mineral density (BMD) provides no information on bone quality, the Trabecular Bone Score (TBS) has been proposed to reflect bone microarchitecture status. The present study was designed to assess prospective changes in TBS and lumbar spine (LS) BMD in postmenopausal women with breast cancer at completion of $\mathrm{Al}$ treatment.

\section{Methods}

B-ABLE is a prospective cohort of 735 women with breast cancer treated with Als according to American Society of Clinical Oncology recommendations: 5 years of Al starting within 6 weeks post-surgery or 1 month after the last cycle of chemotherapy (5y-Al group), or switching to an $\mathrm{Al}$ to complete 5-year therapy after 2-3 years of tamoxifen (pTMX-Al group). Patients with osteoporosis were treated with oral bisphosphonates (BP). TBS and LS-BMD changes at completion of Al therapy were evaluated by Student t-test for paired samples. Pearson correlation coefficients were computed for correlations between LS-BMD and TBS.

\section{$\underline{\text { Results }}$}

Al treatment was completed by 277 women. Of these, 70 (25.3\%) were allocated to BP therapy. The non-BP-treated patients (74.7\%) showed significant decreases in TBS $(-2.94 \%$ in $\mathrm{pTMX}-\mathrm{Al}$ and $-2.93 \%$ in $5 y-A l$ groups) and in LS-BMD (-4.14\% in pTMX-Al and $-2.28 \%$ in $5 \mathrm{y}-\mathrm{Al}$ groups) at the end of Al treatment. In BP-treated patients, TBS remained stable at the end of Al treatment, whereas LS-BMD showed significant increases $(+2.30 \%$ in pTMX-Al and $+5.33 \%$ in $5 \mathrm{y}-\mathrm{Al}$ groups). Moderate associations between TBS and LS-BMD values at baseline and at the end of Al treatment $(r=0.4 ; P<0.001)$ were observed. At the end of treatment, changes in spine BMD and TBS were weakly correlated $(r=0.1, P<0.01)$. 


\section{Conclusions}

Al therapy induces significant decreases in TBS, comparable to BMD loss. BP-treated patients maintained TBS values, whereas BMD increased. Al treatment leads to deterioration of bone microarchitecture, which seems to be attenuated by BP therapy.

\section{KEYWORDS}

Trabecular Bone Score, Aromatase inhibitors, Oral bisphosphonates, Bone mineral density, Tamoxifen, Breast cancer 


\section{INTRODUCTION}

The superiority of aromatase inhibitors (Als) in disease-free survival compared with tamoxifen $(\mathrm{TMX})^{1-3}$ has made them the standard first-line adjuvant therapy among patients with receptorpositive breast cancer. Nevertheless, the routine use of Als has raised concerns about their adverse effects, threatening treatment adherence ${ }^{4-6}$. A profound suppression of estrogen levels is derived from the potent inhibition of the aromatase enzyme caused by these drugs ${ }^{7,8}$. Altreated women often develop osteoporosis, showing an average bone loss rate twice that of the natural reduction during menopause ${ }^{9}$. These differences can result in a $47 \%$ increased fracture risk with Al use, compared with $\mathrm{TMX}^{10}$.

At present, bone mineral density (BMD) measured by dual X-ray absorptiometry (DXA) is the gold standard surrogate for the diagnosis of osteoporosis based on the WHO criteria ${ }^{11}$. However, it is now recognized that the increased risk of non-traumatic fractures associated with osteoporosis is determined not only by the mineral content but also by bone quality and material properties, such as trabecular microarchitecture, the accumulation of microfractures, a disordered bone remodeling, or the influence of extra-skeletal risk factors ${ }^{12-14}$. Hence, the BMD measurement does not provide information on bone quality, which has been estimated to explain $70-75 \%$ of the variance in bone strength ${ }^{15}$.

The Trabecular Bone Score (TBS) is a new additional texture parameter derived from lumbar spine (LS) DXA that is postulated to reflect bone microarchitecture. ${ }^{16-18}$ TBS measures the rate of local variation in gray levels and is related to the structural condition of bone microarchitecture ${ }^{16-18}$ independently of spine and hip $\mathrm{BMD}^{18}$. Overall, in combination with BMD TBS is thought to increase the number of patients with a well-identified fracture risk ${ }^{18-21}$. A high TBS value means that the bone microarchitecture is dense, well-connected, and fractureresistant.

Until now, the limited number of studies of $\mathrm{Al}$ impact on bone microarchitecture has described a larger decrease in BMD compared with $\mathrm{TBS}^{22-24}$.

Oral bisphosphonates (BP) have been approved as an effective antiresorptive therapy for the prevention and treatment not only of osteoporosis in normal postmenopausal women but also in 
the management of Al-related bone loss $(\mathrm{AIBL})^{25-28}$. Nevertheless, the hypothesis that BPrelated BMD increases may reduce fracture risk remains controversial ${ }^{29,30}$. Previous reports evaluating the skeletal preservation of BP in both healthy ${ }^{31,32}$ and Al-treated ${ }^{24}$ postmenopausal women do not describe remarkable improvements but rather a "positive maintenance" of bone microarchitecture. However, many of them include a small sample of patients. Moreover, observations from randomized controlled trials (RCTs) may differ considerably from real-life clinical practice. Therefore, the current study was designed to assess prospective changes in TBS and BMD in postmenopausal women with breast cancer after completing Al treatment.

\section{METHODS}

\section{$\underline{\text { Participants }}$}

From December 2005 to February 2016, Caucasian postmenopausal women diagnosed with hormone receptor-positive early breast cancer and candidates for Al treatment were consecutively recruited in the B-ABLE cohort. Postmenopausal status was defined as patients $>55$ years old with amenorrhea for more than 12 months, or those $\leq 55$ with levels of luteinizing hormone $>30 \mathrm{mIU} / \mathrm{ml}$ or follicle-stimulating hormone values $>40 \mathrm{mIU} / \mathrm{ml}$. Eligible participants were excluded for a history of any bone disease, rheumatoid arthritis, metabolic or endocrine diseases; prior diagnosis of Paget's bone disease or osteomalacia; concurrent or prior treatment with BP, oral corticosteroids, or any other bone-active drug except tamoxifen.

\section{$\underline{\text { Study design and interventions }}$}

B-ABLE is a prospective, non-selected, observational, clinical cohort study carried out at the Breast Cancer Unit and Bone Metabolism Unit, Hospital del Mar, Barcelona, Spain. Participants were treated with Als (letrozole, exemestane, or anastrozole) according to American Society of Clinical Oncology recommendations ${ }^{33}: 5$ years of $\mathrm{Al}$ starting within 6 weeks post-surgery or 1 month after the last cycle of chemotherapy (5y-Al group) or, alternatively, switching to an $\mathrm{Al}$ after taking tamoxifen for 2 to 3 years, to complete 5 years of hormonal therapy (pTMX-Al group).

Patients were stratified by BMD at LS, femoral neck (FN), and total hip (TH) at the outset of the study, and assigned to the corresponding therapeutic regimen: 1) those with osteoporosis 
[ $T$ score $<-2.5$ ] or with a $T$ score $\leq-2.0$ at any site plus 1 major risk factor or prevalent fragility fractures were allocated to weekly oral risedronate or alendronate therapy (BP-treated patients) and 2) no active antiresorptive therapy in all other patients (non-BP-treated patients). All patients had a BMD assessment every 12 months until the end of $\mathrm{Al}$ therapy. Those who developed osteoporosis during the treatment were immediately offered oral BP treatment and were censored from the study at that point.

$\mathrm{Al}$ and BP treatment adherence was assessed by a specific question by the physician at each follow-up visit. Patients with low adherence (defined as medication possession ratio $<80 \%$ ) were excluded from the study.

All participants were supplemented with calcium and $25(\mathrm{OH})$ vit $\mathrm{D}_{3}$ tablets $(1,000 \mathrm{mg}$ and $800 \mathrm{IU}$ daily, respectively), and those with baseline $25(\mathrm{OH})$ vitD deficiency $(<30 \mathrm{ng} / \mathrm{ml})$ received an additional dose of 16,000 IU of oral calcifediol (HIDROFEROL® FAES FARMA) every 2 weeks.

\section{Variables and measurements}

\section{Bone mineral density}

BMD was measured at the LS (L1-L4), FN, and TH using a DXA densitometer QDR 4500 SL® (Hologic, Waltham, MA, USA), in accordance with manufacturer recommendations. In our department, the in vivo coefficient of variation of this technique ranges from $1.0 \%$ at LS to $1.65 \%$ at $\mathrm{FN}$.

\section{Trabecular Bone Score}

Spine TBS measurements were performed using the TBS software installed on our densitometer (TBS iNsight ${ }^{\circledR}$ v2.1, Med-Imaps, Pessac, France). The TBS is calculated on the basis of the raw data acquired in the DXA scan, evaluating the same regions of measurements as those used for the LS-BMD and without further administration of ionizing radiation to the patient.

\section{Other assessments}

Information on a large number of clinical variables was recorded at the time of enrollment, including age at recruitment, age at menarche and menopause, parity, lactation, previous 
chemotherapy and radiotherapy, adjuvant treatments, weight, height, and plasma levels of 25(OH)vitD.

\section{Ethics approval}

The study protocol was approved by the Parc de Salut Mar Ethics Committee and written informed consent was obtained from all participants after they had read the study information sheet and any questions had been answered.

\section{Statistical analyses}

The primary endpoint was within-subject percentage changes in LS-BMD and TBS by the end of Al therapy. Given the observational nature of our study, the results for BP-treated and nonBP-treated patients are presented separately.

TBS and LS-BMD changes from baseline were evaluated using Student t-test for paired samples (mean $\pm 95 \% \mathrm{Cl}$ ). To account for potential differences between the pTMX-Al and $5 \mathrm{y}-\mathrm{Al}$ groups, both BMD and TBS percent changes and absolute values were examined by independent samples t-test.

By analogy with the three BMD categories, cutoff points for TBS have been previously established by a working group of TBS users from different countries: TBS $\geq 1.350$ is considered to be normal, TBS between 1.200 and 1.350 is considered to be consistent with partially degraded microarchitecture, and TBS $\leq 1.200$ defines degraded microarchitecture ${ }^{34}$. Patient distribution throughout these TBS categories ${ }^{34}$ was studied at baseline and at the end of AI treatment. Potential changes in TBS categories from baseline were assessed by marginal homogeneity tests. Pearson correlation coefficients were computed for correlations between spine BMD and TBS.

All statistical tests defined $\mathrm{P}<0.05$ as significant. These analyses were performed with $\mathrm{R}$ for Windows version 2.15.2 using the foreign, ggplot2, reshape, extrafont, scales, grid and boot packages and SPSS 12.0 . 


\section{RESULTS}

$\underline{\text { Participants }}$

From a total of 735 recruited women in the B-ABLE cohort, 277 (37.7\%) completed Al treatment and were eligible for the analyses. Of these, 70 (25.3\%) were allocated to BP therapy (Fig. 1).

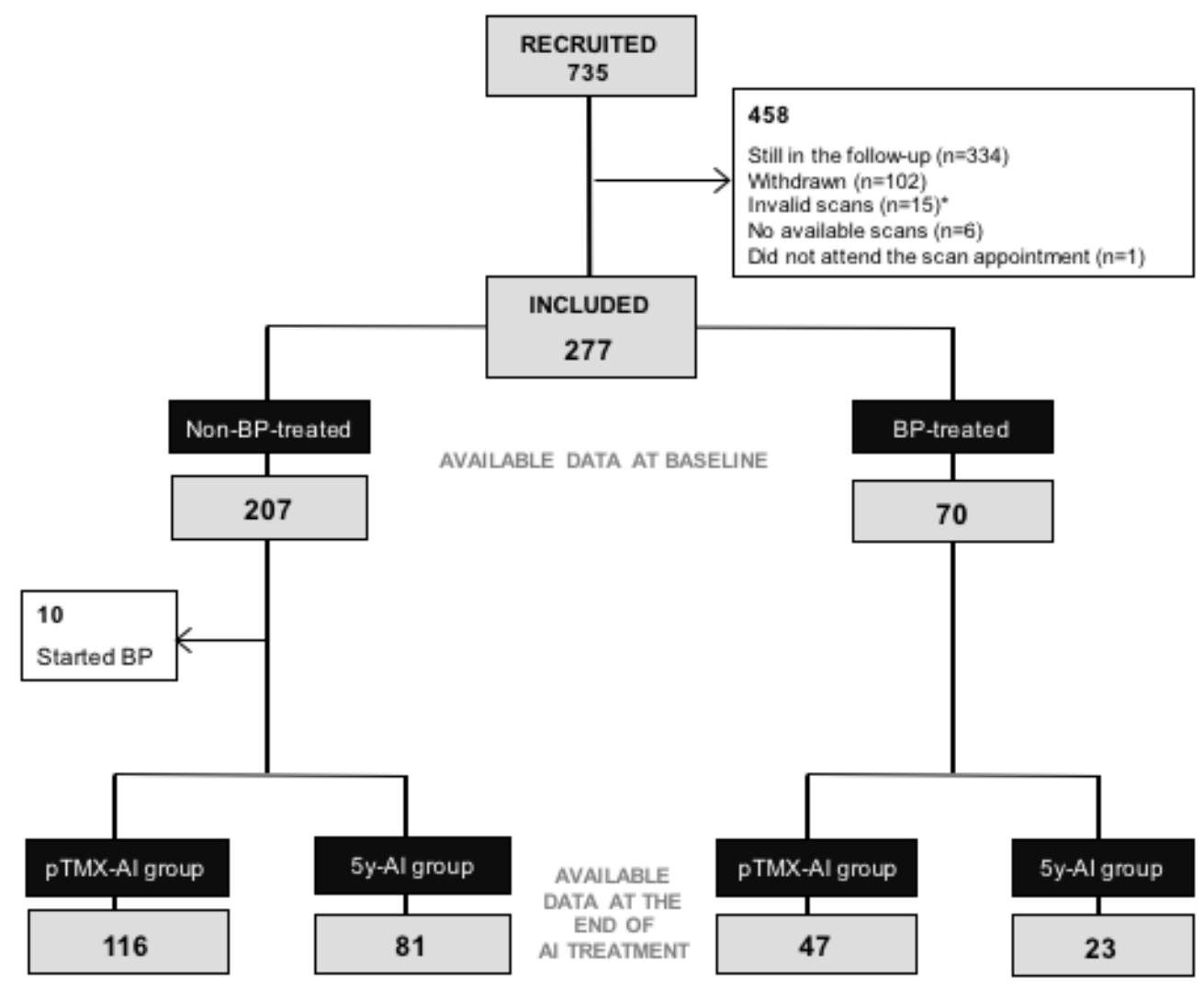

Figure 1: Flow-chart showing the number of patients at baseline and at the end of treatment in the B-ABLE cohort, according to BP therapy. Patients receiving 5 years of $\mathrm{Al}$ constituted the $5 \mathrm{y}$-Al group and those switching to an $\mathrm{Al}$ after taking tamoxifen for 2 to 3 years correspond to the pTMX-Al group. Abbreviations: Al (Aromatase Inhibitors); BP (Oral bisphosphonates). * Scoliosis, arthrodesis, not able to position appropriately for scanning, morbid obesity. 
Baseline patient characteristics are presented in Table 1.

Table 1. Baseline patient characteristics

\section{Patient characteristic}

Mean age (years) \pm (SD)

Mean BMI \pm (SD)

Prior tamoxifen $\mathbf{n}(\%)$

Mean LS TBS (unit less) \pm (SD)

Mean LS BMD $\left(\mathrm{g} / \mathrm{cm}^{2}\right) \pm(\mathrm{SD})$
BP-treated $(n=70 ; 25.3 \%)$

( $n=207 ; 74.7 \%)$
$62.0 \pm 8.3$ $26.9+4.3^{*}$

$29.2 \pm 5.2$
$47(67.1 \%)$

$120(58.0 \%)$
$1.126 \pm 0.114^{*}$ $1.221 \pm 0.130$

$0.790 \pm 0.104^{*}$

Abbreviations: BP (Oral bisphosphonates); SD (Standard deviation); LS (Lumbar spine); BMI (Body mass index); BMD (Bone mineral density); TBS (Trabecular bone score). In t-test compared w ith Non-BP-treated group: ${ }^{*} p<0.001$.

As expected, given the observational nature of our study, the groups differed at baseline: on average, the BP-treated patients had lower BMI, LS-BMD, and LS-TBS than those in the nonBP-treated group $(p<0.001)$. Ten patients in the non-BP-treated group developed osteoporosis during the follow-up. These patients were immediately offered oral BP treatment and they were censored from the study at that point (Fig. 1).

\section{Bone assessment}

Mean percentage changes in LS-BMD and TBS at the end of Al treatment are summarized in Figure 2. 

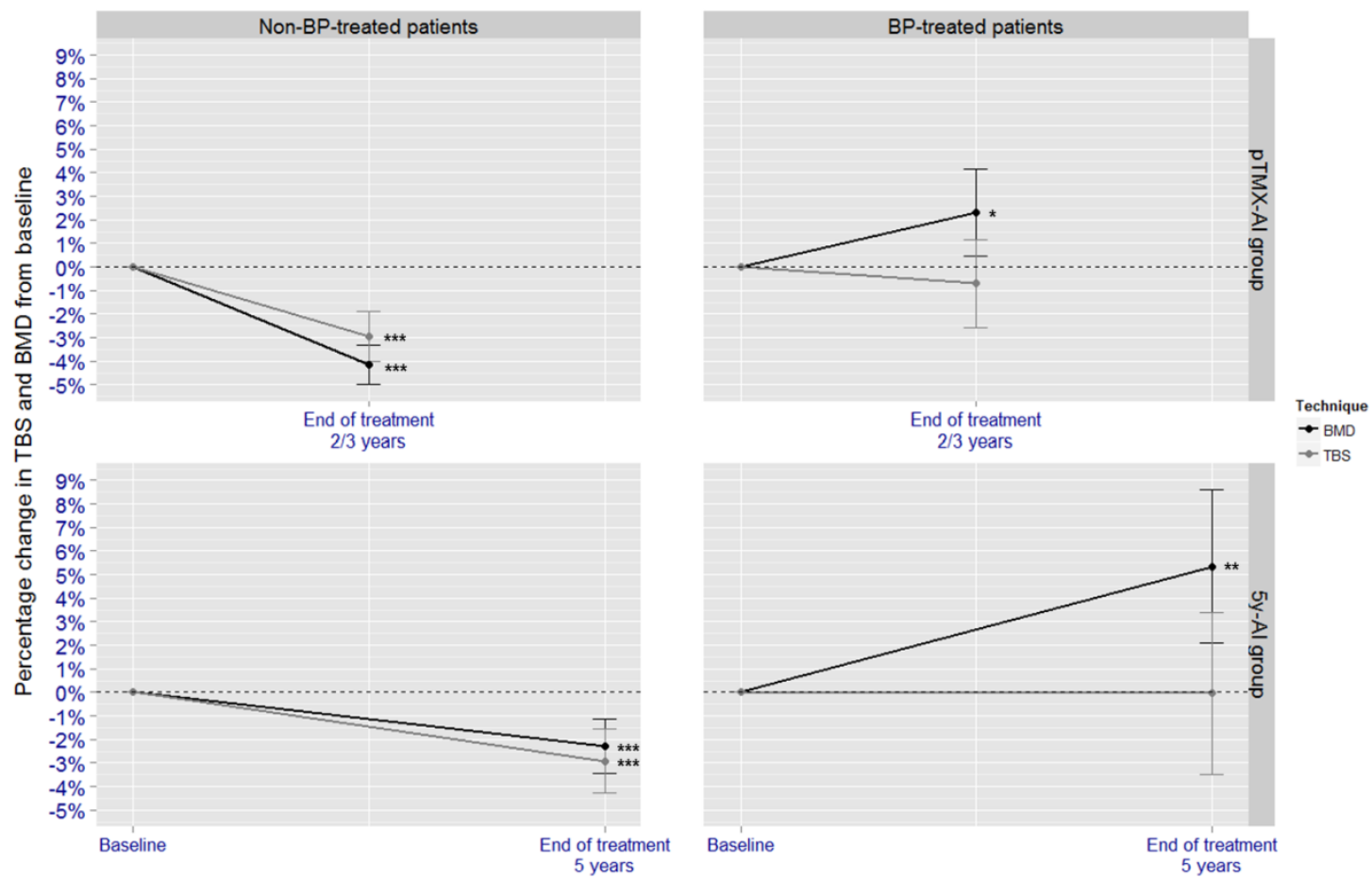

Figure 2: Individual percent change in TBS and LS-BMD at the end of Al treatment according to BP and previous tamoxifen treatment. Mean $\pm 95 \% \mathrm{Cl}$ are reported. In paired t- test from baseline: ${ }^{*}(P<0.05),{ }^{* *}(P<0.01) ;{ }^{* \star *}(P<0.001)$. Abbreviations: Al (Aromatase inhibitors); TBS (Trabecular Bone Score); BMD (Bone mineral density); BP (Oral bisphosphonates).

Absolute TBS and LS-BMD values at baseline and at the end of treatment are detailed in Table 2.

Table 2: Absolute TBS and LS-BMD values (Mean \pm SD) in postmenopausal woman with breast cancer at baseline and at the end of
Al treatment according to BP treatment and previous TMX use.
\begin{tabular}{c|c|c|c|c|c}
\hline \multirow{2}{*}{ Measure } & \multirow{2}{*}{ Lenght of follow-up } & \multicolumn{2}{c}{ Non-BP-treated patients } & \multicolumn{2}{c}{ BP-treated patients } \\
\cline { 3 - 6 } & & pTMX-Al & 5 y-Al & pTMX-Al & 5 y-Al \\
\hline \multirow{2}{*}{ TBS } & Baseline & $1.239 \pm 0.130$ & $1.203 \pm 0.124$ & $1.150 \pm 0.104$ & $1.078 \pm 0.122^{*}$ \\
\cline { 2 - 6 } & End of treatment & $1.200 \pm 0.119$ & $1.165 \pm 0.116^{*}$ & $1.141 \pm 0.115$ & $1.075 \pm 0.133^{*}$ \\
\hline \multirow{2}{*}{ BMD } & Baseline & $0.963 \pm 0.101$ & $0.965 \pm 0.106$ & $0.808 \pm 0.103$ & $0.755 \pm 0.098^{*}$ \\
\cline { 2 - 5 } & End of treatment & $0.923 \pm 0.105$ & $0.942 \pm 0.110$ & $0.825 \pm 0.098$ & $0.793 \pm 0.103$
\end{tabular}

Abbreviations: LS-BMD (Lumbar spine-Bone mineral density); TBS (Trabecular bone score); SD (Standard deviation); BP (Oral bisphosphonates); TMX (Tamoxifen); Al (Aromatase inhibitors). In t-test compared with pTMX-Al: ${ }^{*}(\mathrm{p}<0.05)$. 


\section{Non-BP-treated patients}

At the end of Al treatment, significant TBS decrease was observed in both groups: pTMX-Al, $2.94 \%\left(-0.039 \mathrm{~g} / \mathrm{cm}^{2}\right.$ [95\%Cl: -0.051 to -0.027 ]; $\left.\mathrm{P}<0.001\right)$ and $5 \mathrm{y}-\mathrm{Al},-2.93 \%\left(-0.038 \mathrm{~g} / \mathrm{cm}^{2}\right.$ [95\% Cl: -0.054 to -0.022$]$; $\mathrm{P}<0.001$ ) (Fig. 2). An inter-group comparison did not reveal significant differences in TBS decreases between the pTMX-AI and 5y-Al groups.

Significant decreases in LS-BMD were also detected in both groups: pTMX-AI, $-4.14 \%(-0.040$ $\mathrm{g} / \mathrm{cm}^{2}$ [95\%Cl: -0.048 to -0.032 ]; $\left.\mathrm{P}<0.001\right)$ and $5 \mathrm{y}-\mathrm{Al},-2.28 \%\left(-0.023 \mathrm{~g} / \mathrm{cm}^{2}[95 \% \mathrm{Cl}:-0.034\right.$ to 0.011]; $\mathrm{P}<0.001)$. In this case, the pTMX-Al group experienced significantly greater LS-BMD decline $(-4.14 \%)$, compared to the $5 y-A l$ group $(-2.28 \% ; \mathrm{P}<0.05)$.

No significant difference was found in baseline TBS between the pTMX-Al and 5y-Al groups, whereas TBS values at the end of treatment were lower in the 5y-Al group $(P<0.05)$. There were no significant differences in LS-BMD between the two groups at baseline or at the end of treatment (Table 2).

BP-treated patients

The TBS remained stable in both pTMX-Al and 5y-Al groups at the end of treatment (Fig.2). Compared to baseline, there was a significant increase in LS-BMD at the end of treatment in both groups, reaching $+2.30 \%\left(0.016 \mathrm{~g} / \mathrm{cm}^{2}\right.$ [95\% $\mathrm{Cl}$ : 0.001 to 0.031 ]; $\left.\mathrm{P}<0.05\right)$ in the pTMX-AI group and $+5.33 \%\left(0.038 \mathrm{~g} / \mathrm{cm}^{2}\right.$ [95\%Cl: 0.017 to 0.059$]$; $\left.\mathrm{P}<0.01\right)$ in the $5 \mathrm{y}-\mathrm{Al}$ group. However, the difference between the groups in these LS-BMD gains was not significant.

Assessment of absolute measures revealed greater TBS values in the pTMX-Al group at baseline and at the end of treatment, compared with the 5y-Al group $(P<0.05)$. Although baseline LS-BMD values were higher in the PTMX-AI group $(P<0.05)$, the values were similar in both groups at the end of treatment (Table 2).

Changes in TBS categories

Analysis of TBS range revealed significant changes in the frequency distribution of non-BPtreated patients from baseline to the end of $A l$ treatment $(P<0.01)$ (Fig.3). 


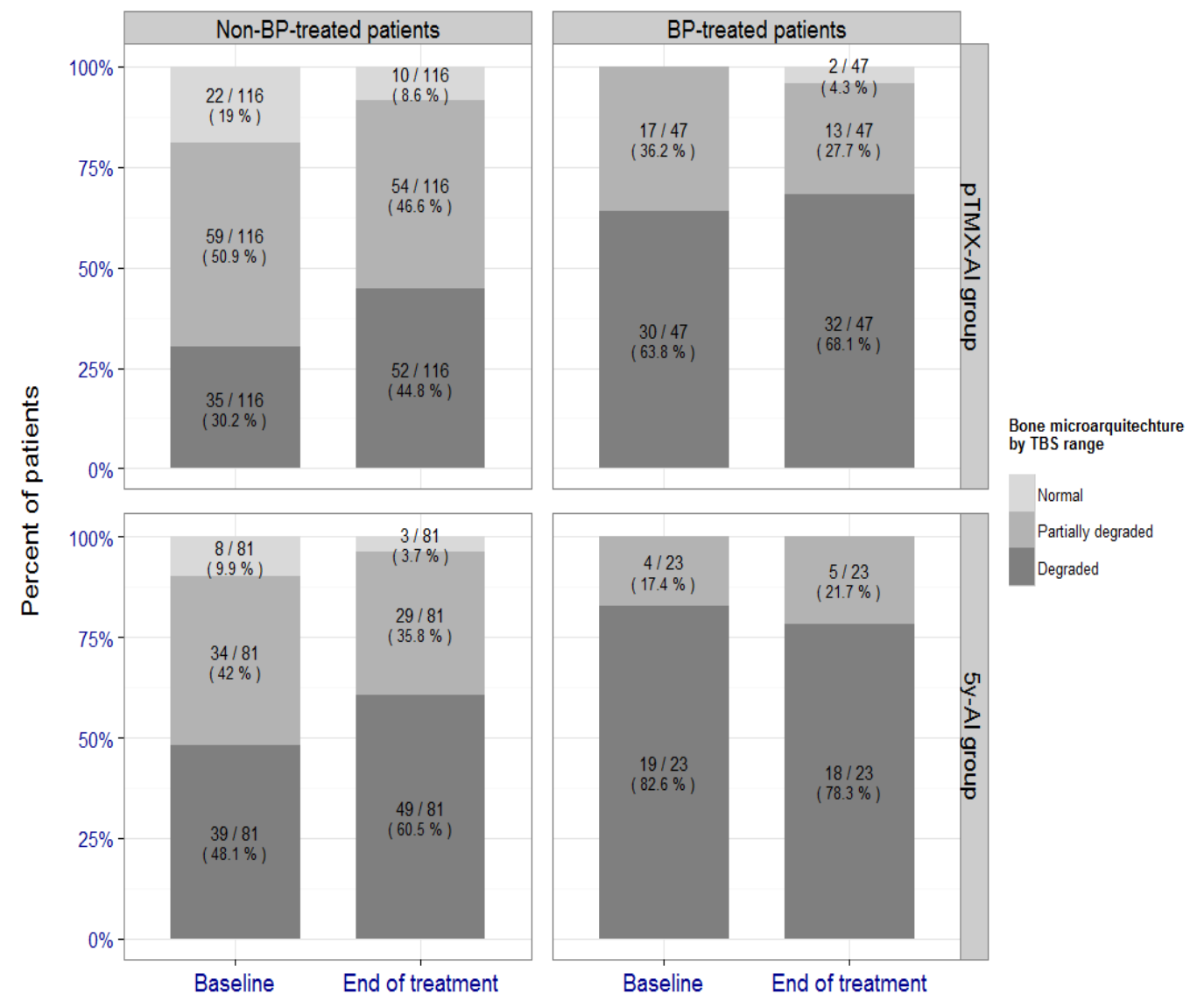

Figure 3: Percentages of patient distribution across TBS range at baseline and at the end of treatment, according to BP and previous tamoxifen treatment. Abbreviations: TBS (Trabecular Bone Score); BP (oral bisphosphonates).

The number of patients with degraded microarchitecture increased by $32.6 \%$ in the pTMX-AI group and by $20.5 \%$ in the $5 \mathrm{y}-\mathrm{Al}$ group; in contrast, the number of patients falling within the normal range decreased by $55 \%$ and $62.5 \%$ at the end of treatment, respectively. On the other hand, no significant differences in frequency distribution throughout TBS categories in BPtreated patients were detected in either group (Fig.3). Figure 4 shows results exclusively for patients who were recategorized from baseline to the end of treatment. 


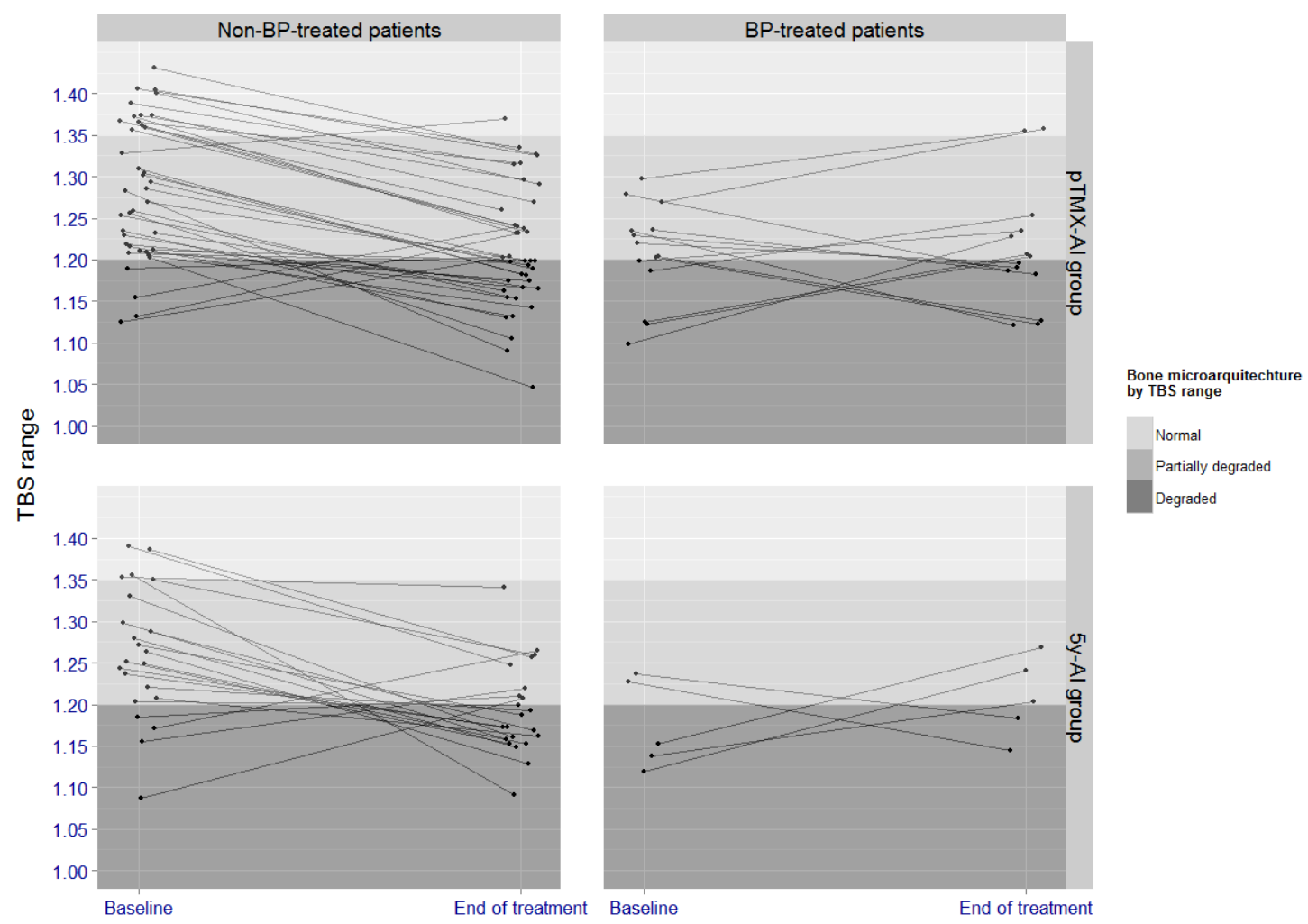

Figure 4: Patients who changed TBS category from baseline to end of treatment according to BP treatment and previous tamoxifen use. Abbreviations: TBS (Trabecular Bone Score); BP (oral bisphosphonates).

At the end of Al therapy, 52/197 (26.4\%) non-BP-treated patients had a decrease of one TBS category: $18 / 197$ (9.1\%) patients shifted from the normal to the partially degraded category and $34 / 197$ (17.3\%) from partially degraded to degraded microarchitecture. Only 9/197 (4.6\%) nonBP-treated patients moved to a higher TBS category and 1 patient $(0.5 \%)$ made an exceptional shift of two categories, from normal to degraded microarchitecture. In the case of BP-treated women, $10 / 70(14.2 \%)$ patients moved up one category, while $9 / 70(12.8 \%)$ fell from the partially degraded to the degraded range.

\section{BMD and TBS correlation}

Moderate associations between TBS and LS-BMD values at baseline and at the end of AI treatment $(r=0.4 ; P<0.001)$ have been observed. At the end of treatment, changes in spine BMD and TBS were weakly correlated $(r=0.1, P<0.01)$. 


\section{DISCUSSION}

To our knowledge this is the first longitudinal cohort study to assess changes in TBS at the end of Al-therapy in postmenopausal women with hormone receptor-positive breast cancer. Both TBS and LS-BMD decreased significantly in non-BP-treated women. BP-treated patients showed significant increases in BMD but not in TBS. TBS and BMD reductions were more pronounced in PTMX-Al group than those in 5y-Al group. In spite of this, absolute TBS values at Al-treatment completion were similar for both groups.

Although BMD is the reference standard for osteoporosis detection and follow-up, as well us for fracture prediction, it remains subject to a set of constraints. Unfortunately, BMD does not evaluate other factors influencing bone strength, such as microarchitectural deterioration, and disproportionately evaluates cortical bone (representing $80 \%$ of bone volume), which has a relatively slow rate of turnover ${ }^{35}$. These limitations can underestimate the sudden changes that might occur within the trabecular compartment. The majority of patients who sustain fragility fractures have a T-score $>-2.5^{36,37}$, there is thus an overlap between BMD values in patients with and without osteoporotic fractures ${ }^{38,39}$. Therefore, the current definition of osteoporosis includes both a decrease in BMD and impaired bone microarchitecture resulting in increased bone fragility, defined as reduced connectivity of the trabecular bone structure and thinning and increased porosity of the cortical bone.

Skeletal microstructure can be assessed by techniques such as histomorphometric analysis of the transiliac crest bone biopsy, high-resolution peripheral quantitative computed tomography $(\mathrm{HRpQCT})^{40}$, flat-panel volume $\mathrm{CT}^{41}$, and magnetic resonance imaging (MRI) ${ }^{42}$. However, these procedures are either invasive or not routinely available, being limited to research purposes. TBS is a novel complementary technique for fracture risk assessment ${ }^{43}$ that has been correlated with connectivity of bone trabeculae, trabecular bone volume, and compressive stiffness $^{18,44}$. TBS is able to distinguish between DXA scans with similar bone density ${ }^{18,45}$, capturing roughly one third of fractures misclassified using BMD alone ${ }^{32}$.

In the present study, significant TBS decreases were observed in non-BP-treated patients at the end of Al treatment, both in the pTMX-Al (-2.94\%) and 5y-Al (-2.93\%) groups. Assuming 
linearity, these reductions in TBS would correspond to mean annual rates of $1.2 \%$ (2-3 years of $\mathrm{Al}$ ) and $0.6 \%$ (5 years of $\mathrm{Al})$. The age-adjusted TBS curves reported in previous cross-sectional studies show much lower annual decline rates, even for the group of patients aged 65 years and older, in which the decrease accelerates to $0.5 \%$ per year $(0.006)^{46,47}$. In RCTs evaluating the effect of antiresorptive agents on TBS in postmenopausal women, annual reduction rates for the placebo groups were similar to our results ${ }^{31,32}$. Regarding TBS pre-stablished categories ${ }^{34}$, a large number of non-BP-treated women (approximately 30\%) decreased by one category. Hence, the greatest number of patients belonged to the degraded microarchitecture category at the end of $\mathrm{Al}$ treatment.

All this suggests that Als substantially affect bone microarchitecture. Consistent with this view, Prasad et al described mean TBS changes of $-2.35 \%$ at 2 years of $\mathrm{Al}$ treatment ${ }^{48}$ and Kalder at al found TBS reductions of $-2.3 \%$ in exemestane-treated women over the same timeframe ${ }^{23}$. Similarly, Al-treated patients in another retrospective cohort study experienced TBS decreases of $-2.1 \%$ at a mean follow-up of 2.3 years $^{22}$.

The marked reduction in estrogen levels caused by Al exacerbates the increased bone resorption and excess fracture risk induced by menopause ${ }^{2}$. In our cohort, women without BP treatment showed LS-BMD decreases of $-4.14 \%$ in the PTMX-AI group and $-2.28 \%$ in the $5 \mathrm{y}-\mathrm{Al}$ group by the end of $\mathrm{Al}$ treatment. Large adjuvant trials have described higher bone loss rates associated with Al treatment. For example, the ATAC trial (Arimidex, Tamoxifen, Alone or in Combination) detected LS bone loss rates from baseline to 5 years of anastrozol of $-6.08 \%{ }^{49}$. Likewise, RCTs examining Al use after tamoxifen therapy have reported BMD reductions at LS of $-4.16 \%\left(\right.$ IES trial $\left.{ }^{50}\right)$ and $-5.35 \%\left(\mathrm{MA}-17\right.$ trial $\left.^{51}\right)$.

Overall, the average bone loss in our population was lower than previously reported. Differences in some characteristics, such as initial BMD values, may contribute to this outcome. In this sense, most of the trials mentioned report higher BMD values at baseline than those observed in our cohort, giving rise to the regression-to-the-mean bias. Moreover, the B-ABLE cohort is subject to strict monitoring not only of BMD but also of $25(\mathrm{OH})$ vitD and calcium levels. Patients in our study receive higher $25(\mathrm{OH})$ vitD supplementation ${ }^{52,53}$ than the Institute of 
Medicine recommendations ${ }^{54}$. Conversely, the unavailability of TBS values at the end of $\mathrm{Al}$ treatment in previous studies precludes a direct comparison with our data.

In the current analysis, women in the pTMX-Al group showed greater individual reductions in $\mathrm{BMD}$ at $\mathrm{Al}$ completion, compared to the $5 \mathrm{y}-\mathrm{Al}$ group $(-4.14 \%$ vs. $-2.28 \%)$. These results are similar to those we previously described in an analysis of Al effects at 3 years of follow-up ${ }^{55}$. Analogous findings have been detected for TBS: despite a similar reduction in TBS values at AItreatment completion $(-2.93 \%$ vs. $-2.94 \%)$, we must consider that patients in the pTMX-Al group have been treated for a shorter time with Al. Tamoxifen has been shown to have partial estrogen-agonist actions in bone, exerting beneficial effects such as reducing bone resorption and stimulating bone formation in postmenopausal women with breast cancer ${ }^{56}$. Moreover, Kalder et al demonstrated that tamoxifen induced significant sustained increases in TBS, possibly indicating a compensation and/or stabilization of bone texture parameters ${ }^{23}$. The rebound effect of tamoxifen could be the underlying factor contributing to this phenomenon: the early increases observed not only cease with interruption of tamoxifen ${ }^{57}$, but are also associated with a profound bone loss when switching to $\mathrm{Al}^{58}$. Despite these findings, the pTMX-Al group in our study did not have lower mean TBS or BMD absolute values at the end of treatment, compared with the 5y-Al group.

Patients with osteoporosis or with osteopenia at high risk of fracture at baseline were allocated to BP therapy in our study. Although LS-BMD increased in both groups (pTMX-Al, $+2.30 \%$; $5 y$ Al, 5.33\%), the TBS remained stable from baseline to the end of Al treatment. The increases in BMD values for patients in the $5 \mathrm{y}-\mathrm{Al}$ group is within the range of increases reported in large trials evaluating the effects of $\mathrm{BP}$ on $\mathrm{AlBL}^{59,60}$. The lack of statistical significance in $\mathrm{BMD}$ variation between groups $(+2.30 \%$ vs. $+5.33 \%)$ may be due to the reduced sample size.

Overall, TBS behavior in our cohort was comparable to the results obtained in the longitudinal study of Krieg et al, evaluating the effects of antiresorptive agents in TBS of older women: although TBS mimicked BMD decreases in individuals without BP treatment, it seemed to be far less responsive than BMD to osteoporosis therapy ${ }^{31}$. This is also applicable in RCTs monitoring the effectiveness of BP treatment, which have detected greater increases in BMD than in $\mathrm{TBS}^{24,32,48}$. Moreover, the frequency of distribution throughout TBS categories for BP-treated patients was the same at baseline and at the end of Al treatment, with almost the same number 
of individuals increasing (10 patients, 14.2\%) and decreasing (9 patients, 12.8\%) in TBS category.

In summary, antiresorptive therapy is expected to provide global maintenance of bone microarchitecture rather than major improvement. Although BPs have improved several bone parameters in some studies, the greatest improvements have been detected in patients who did not receive an $\mathrm{Al}^{61}$. The uncertainty about whether BP treatment prevents fractures in early breast cancer patients ${ }^{62}$ may be evidence that use of an $\mathrm{Al}$ can induce changes in bone microarchitecture that are more difficult to counteract by antiosteoporotic doses of BPs. The weak correlation between the changes in TBS and BMD at 5-year follow-up supports the different bone properties measured by these two techniques.

The present study has some limitations due to the prospective nature of the analysis and the relatively small sample size. Moreover, this is not a randomized controlled study, but a prospective cohort based on real-life clinical practice. Thus, in order to firmly conclude that BP therapy was the cause of the observed BMD and TBS behavior in BP-treated patients, a control group of women with low baseline BMD and without BP treatment would be required. Obviously, this is not feasible for ethical reasons. Another limitation is that the evaluation of $\mathrm{Al}$ and $\mathrm{BP}$ treatment adherence was only assessed by physician questionnaire. A strength of our study is that it was performed when Al treatment was completed and provides insights about the bone status of women with breast cancer at the end of their adjuvant therapy in real-life clinical practice.

\section{CONCLUSIONS}

In this prospective cohort study, Al treatment decreased TBS and LS-BMD to a similar extent. On the other hand, maintenance of TBS, rather than major improvement, was observed in BPtreated patients. Al treatment leads to bone microarchitecture deterioration, which seems to be partially attenuated by BPs. Larger studies are needed to determine whether the observed TBS changes are correlated with changes in fracture risk.

\section{DECLARATION OF INTERESTS}

The authors state that they have no conflicts of interest. 


\section{FUNDING}

This work was supported by the Red Temática de Investigación Cooperativa en Envejecimiento y Fragilidad (RETICEF; RD12/0043/0022), and the Grant FIS PI10/01464 and PI13/00444

(Carlos III Health Institute, Science and Innovation Ministry); Grants from the Generalitat de Catalunya (DIUE 2014 SGR 775) and FEDER funds have supported this study.

\section{ACKNOWLEDGMENTS}

The authors thank Elaine M. Lilly, Ph.D, for helpful advice and critical reading of the manuscript. 


\section{REFERENCES}

1. Coates AS, Keshaviah A, Thurlimann B, et al: Five years of letrozole compared with tamoxifen as initial adjuvant therapy for postmenopausal women with endocrineresponsive early breast cancer: update of study BIG 1-98. J Clin Oncol 25:486-92, 2007

2. Howell A, Cuzick J, Baum M, et al: Results of the ATAC (Arimidex, Tamoxifen, Alone or in Combination) trial after completion of 5 years' adjuvant treatment for breast cancer. Lancet 365:60-2, 2005

3. Coombes RC, Kilburn LS, Snowdon CF, et al: Survival and safety of exemestane versus tamoxifen after 2-3 years' tamoxifen treatment (Intergroup Exemestane Study): a randomised controlled trial. Lancet 369:559-70, 2007

4. Servitja S, Martos T, Rodriguez Sanz M, et al: Skeletal adverse effects with aromatase inhibitors in early breast cancer: evidence to date and clinical guidance. Ther Adv Med Oncol 7:291-6, 2015

5. Henry NL, Azzouz F, Desta Z, et al: Predictors of aromatase inhibitor discontinuation as a result of treatment-emergent symptoms in early-stage breast cancer. J Clin Oncol 30:936-42, 2012

6. Hadji P, Jackisch C, Bolten W, et al: COMPliance and Arthralgia in Clinical Therapy: the COMPACT trial, assessing the incidence of arthralgia, and compliance within the first year of adjuvant anastrozole therapy. Ann Oncol 25:372-7, 2014

7. Geisler J, Haynes B, Anker G, et al: Influence of letrozole and anastrozole on total body aromatization and plasma estrogen levels in postmenopausal breast cancer patients evaluated in a randomized, cross-over study. J Clin Oncol 20:751-7, 2002

8. Geisler J, King N, Anker G, et al: In vivo inhibition of aromatization by exemestane, a novel irreversible aromatase inhibitor, in postmenopausal breast cancer patients. Clin Cancer Res 4:2089-93, 1998

9. Hadji P: Aromatase inhibitor-associated bone loss in breast cancer patients is distinct from postmenopausal osteoporosis. Critical Reviews in Oncology/Hematology 69:7382,2009

10. Amir E, Seruga B, Niraula S, et al: Toxicity of adjuvant endocrine therapy in postmenopausal breast cancer patients: a systematic review and meta-analysis. J Natl Cancer Inst 103:1299-309, 2011

11. Assessment of fracture risk and its application to screening for postmenopausal osteoporosis. Report of a WHO Study Group. World Health Organ Tech Rep Ser 843:1-129, 1994

12. Bouxsein ML: Determinants of skeletal fragility. Best Pract Res Clin Rheumatol 19:897-911, 2005

13. Brandi ML: Microarchitecture, the key to bone quality. Rheumatology (Oxford) 48 Suppl 4:iv3-8, 2009

14. Rice JC, Cowin SC, Bowman JA: On the dependence of the elasticity and strength of cancellous bone on apparent density. J Biomech 21:155-68, 1988

15. Ammann P, Rizzoli R: Bone strength and its determinants. Osteoporos Int 14 Suppl 3:S13-8, 2003

16. Pothuaud L, Carceller P, Hans D: Correlations between grey-level variations in 2D projection images (TBS) and 3D microarchitecture: Applications in the study of human trabecular bone microarchitecture. Bone 42:775-787, 2008

17. Winzenrieth R, Michelet F, Hans D: Three-Dimensional (3D) Microarchitecture Correlations with 2D Projection Image Gray-Level Variations Assessed by Trabecular Bone Score Using High-Resolution Computed Tomographic Acquisitions: Effects of Resolution and Noise. Journal of Clinical Densitometry 16:287-296, 2013

18. Hans D, Barthe N, Boutroy S, et al: Correlations Between Trabecular Bone Score, Measured Using Anteroposterior Dual-Energy X-Ray Absorptiometry Acquisition, and 3- 
Dimensional Parameters of Bone Microarchitecture: An Experimental Study on Human Cadaver Vertebrae. Journal of Clinical Densitometry 14:302-312, 2011

19. Boutroy S, Hans D, Sornay-Rendu E, et al: Trabecular bone score improves fracture risk prediction in non-osteoporotic women: the OFELY study. Osteoporos Int 24:77-85, 2013

20. Rabier B, Heraud A, Grand-Lenoir C, et al: A multicentre, retrospective casecontrol study assessing the role of trabecular bone score (TBS) in menopausal Caucasian women with low areal bone mineral density (BMDa): Analysing the odds of vertebral fracture. Bone 46:176-81, 2010

21. Del Rio LM, Winzenrieth R, Cormier C, et al: Is bone microarchitecture status of the lumbar spine assessed by TBS related to femoral neck fracture? A Spanish case-control study. Osteoporos Int 24:991-8, 2013

22. Pedrazzoni M, Casola A, Verzicco I, et al: Longitudinal changes of trabecular bone score after estrogen deprivation: effect of menopause and aromatase inhibition. $J$ Endocrinol Invest 37:871-4, 2014

23. Kalder M, Hans D, Kyvernitakis I, et al: Effects of Exemestane and Tamoxifen treatment on bone texture analysis assessed by TBS in comparison with bone mineral density assessed by DXA in women with breast cancer. J Clin Densitom 17:66-71, 2014

24. Kalder M, Kyvernitakis I, Albert US, et al: Effects of zoledronic acid versus placebo on bone mineral density and bone texture analysis assessed by the trabecular bone score in premenopausal women with breast cancer treatment-induced bone loss: results of the ProBONE II substudy. Osteoporos Int 26:353-60, 2015

25. Reginster J, Minne HW, Sorensen $\mathrm{OH}$, et al: Randomized trial of the effects of risedronate on vertebral fractures in women with established postmenopausal osteoporosis. Vertebral Efficacy with Risedronate Therapy (VERT) Study Group. Osteoporosis International 11:83-91, 2000

26. Brufsky AM, Bosserman LD, Caradonna RR, et al: Zoledronic acid effectively prevents aromatase inhibitor-associated bone loss in postmenopausal women with early breast cancer receiving adjuvant letrozole: Z-FAST study 36-month follow-up results. Clin Breast Cancer 9:77-85, 2009

27. Eidtmann $\mathrm{H}$, de Boer $\mathrm{R}$, Bundred $\mathrm{N}$, et al: Efficacy of zoledronic acid in postmenopausal women with early breast cancer receiving adjuvant letrozole: 36 -month results of the ZO-FAST Study. Ann Oncol 21:2188-94, 2010

28. Van Poznak C, Hannon RA, Mackey JR, et al: Prevention of aromatase inhibitorinduced bone loss using risedronate: the SABRE trial. J Clin Oncol 28:967-75, 2010

29. Valachis A, Polyzos NP, Georgoulias V, et al: Lack of evidence for fracture prevention in early breast cancer bisphosphonate trials: a meta-analysis. Gynecol Oncol $117: 139-45,2010$

30. Coleman R, Powles T, Paterson A, et al: Adjuvant bisphosphonate treatment in early breast cancer: meta-analyses of individual patient data from randomised trials. Lancet 386:1353-61, 2015

31. Krieg MA, Aubry-Rozier B, Hans D, et al: Effects of anti-resorptive agents on trabecular bone score (TBS) in older women. Osteoporos Int 24:1073-8, 2013

32. Popp AW, Guler S, Lamy O, et al: Effects of zoledronate versus placebo on spine bone mineral density and microarchitecture assessed by the trabecular bone score in postmenopausal women with osteoporosis: a three-year study. J Bone Miner Res 28:449-54, 2013

33. Winer EP, Hudis C, Burstein HJ, et al: American Society of Clinical Oncology Technology Assessment on the Use of Aromatase Inhibitors As Adjuvant Therapy for Postmenopausal Women With Hormone Receptor-Positive Breast Cancer: Status Report 2004. Journal of Clinical Oncology 23:619-629, 2005 
34. Cormier C, Lamy O, Poriau S: TBS in routine clinical practice: proposals of use [Internet]. Plan-les-Outes, Switzerland: Medimaps Group; 2012; Available from: http://www.medimapsgroup.com/upload/MEDIMAPS-UK-WEB.pdf.

35. Sawada K, Morishige K, Ohmichi M, et al: Peripheral quantitative computed tomography ( $\mathrm{pQCT}$ ) is useful for monitoring bone mineral density of the patients who receive hormone replacement therapy. Maturitas 56:343-9, 2007

36. Cranney A, Jamal SA, Tsang JF, et al: Low bone mineral density and fracture burden in postmenopausal women. Cmaj 177:575-80, 2007

37. Siris ES, Chen YT, Abbott TA, et al: Bone mineral density thresholds for pharmacological intervention to prevent fractures. Arch Intern Med 164:1108-12, 2004

38. Siris ES, Miller PD, Barrett-Connor E, et al: Identification and fracture outcomes of undiagnosed low bone mineral density in postmenopausal women: results from the National Osteoporosis Risk Assessment. Jama 286:2815-22, 2001

39. Hordon LD, Raisi M, Aaron JE, et al: Trabecular architecture in women and men of similar bone mass with and without vertebral fracture: I. two-dimensional histology. Bone 27:271-276, 2000

40. Boutroy S, Bouxsein ML, Munoz F, et al: In vivo assessment of trabecular bone microarchitecture by high-resolution peripheral quantitative computed tomography. J Clin Endocrinol Metab 90:6508-15, 2005

41. Genant HK, Engelke K, Prevrhal S: Advanced CT bone imaging in osteoporosis. Rheumatology 47, 2008

42. Krug R, Carballido-Gamio J, Banerjee $S$, et al: In vivo ultra-high-field magnetic resonance imaging of trabecular bone microarchitecture at $7 \mathrm{~T}$. Journal of Magnetic Resonance Imaging 27:854-859, 2008

43. Harvey NC, Glüer CC, Binkley N, et al: Trabecular bone score (TBS) as a new complementary approach for osteoporosis evaluation in clinical practice. Bone 78:216-224, 2015

44. Roux JP, Wegrzyn J, Boutroy S, et al: The predictive value of trabecular bone score (TBS) on whole lumbar vertebrae mechanics: an ex vivo study. Osteoporos Int 24:245560,2013

45. Pothuaud L, Barthe N, Krieg M-A, et al: Evaluation of the Potential Use of Trabecular Bone Score to Complement Bone Mineral Density in the Diagnosis of Osteoporosis: A Preliminary Spine BMD-Matched, Case-Control Study. Journal of Clinical Densitometry 12:170-176, 2009

46. Dufour R, Winzenrieth R, Heraud A, et al: Generation and validation of a normative, age-specific reference curve for lumbar spine trabecular bone score (TBS) in French women. Osteoporos Int 24:2837-46, 2013

47. Simonelli C, Leib E, Mossman N, et al: Creation of an age-adjusted, dual-energy $\mathrm{x}$-ray absorptiometry-derived trabecular bone score curve for the lumbar spine in non-Hispanic US White women. J Clin Densitom 17:314-9, 2014

48. Prasad C, Perera S, Greenspan SL: Risedronate May Preserve Bone Microarchitecture in Breast Cancer Survivors on Aromatase Inhibitors, Osteoporosis, 2016, pp FRI-254-FRI-254

49. Eastell R, Adams JE, Coleman RE, et al: Effect of Anastrozole on Bone Mineral Density: 5-Year Results From the Anastrozole, Tamoxifen, Alone or in Combination Trial 18233230. Journal of Clinical Oncology 26:1051-1057, 2008

50. Coleman RE, Banks LM, Girgis SI, et al: Reversal of skeletal effects of endocrine treatments in the Intergroup Exemestane Study. Breast Cancer Research and Treatment 124:153-161, 2010

51. Perez EA, Josse RG, Pritchard KI, et al: Effect of Letrozole Versus Placebo on Bone Mineral Density in Women With Primary Breast Cancer Completing 5 or More Years of 
Adjuvant Tamoxifen: A Companion Study to NCIC CTG MA.17. Journal of Clinical Oncology 24:3629-3635, 2006

52. Prieto-Alhambra D, Servitja S, Javaid MK, et al: Vitamin D threshold to prevent aromatase inhibitor-related bone loss: the B-ABLE prospective cohort study. Breast Cancer Res Treat 133:1159-67, 2012

53. Rodriguez-Sanz M, Garcia-Giralt N, Prieto-Alhambra D, et al: CYP11A1 expression in bone is associated with aromatase inhibitor-related bone loss. J Mol Endocrinol 55:69-79, 2015

54. Ross AC, Manson JE, Abrams SA, et al: The 2011 Report on Dietary Reference Intakes for Calcium and Vitamin D from the Institute of Medicine: What Clinicians Need to Know. The Journal of Clinical Endocrinology and Metabolism 96:53-58, 2011

55. Rodriguez-Sanz M, Prieto-Alhambra D, Servitja S, et al: Al-related BMD variation in actual practice conditions: a prospective cohort study. Endocr Relat Cancer 24:160025,2016

56. Resch A, Biber E, Seifert $M$, et al: Evidence that tamoxifen preserves bone density in late postmenopausal women with breast cancer. Acta Oncol 37:661-4, 1998

57. McCaig FM, Renshaw L, Williams L, et al: A study of the effects of the aromatase inhibitors anastrozole and letrozole on bone metabolism in postmenopausal women with estrogen receptor-positive breast cancer. Breast Cancer Res Treat 119:643-51, 2010

58. McCaig FM, Renshaw L, Williams L, et al: A study of the effects of the aromatase inhibitors anastrozole and letrozole on bone metabolism in postmenopausal women with estrogen receptor-positive breast cancer. Breast Cancer Research and Treatment 119:643-651, 2009

59. Brufsky AM, Harker WG, Beck JT, et al: Final 5-year results of Z-FAST trial. Cancer 118:1192-1201, 2012

60. Coleman R, de Boer R, Eidtmann $\mathrm{H}$, et al: Zoledronic acid (zoledronate) for postmenopausal women with early breast cancer receiving adjuvant letrozole (ZO-FAST study): final 60-month results. Annals of Oncology 24:398-405, 2013

61. van Londen GJ, Perera S, Vujevich KT, et al: The effect of risedronate on hip structural geometry in chemotherapy-induced postmenopausal women with or without use of aromatase inhibitors: A 2-year trial. Bone 46:655-659, 2010

62. Luck AA, Evans AJ, Green AR, et al: The influence of basal phenotype on the metastatic pattern of breast cancer. Clin Oncol (R Coll Radiol) 20:40-5, 2008 